\section{(1)}

CrossMark

\title{
Promoting respiratory public health through epigenetics research: an ERS Environment Health Committee workshop report
}

\author{
Erik Melén (10 1,2,3, Robert Barouki ${ }^{4,5,6}$, Maeve Barry ${ }^{7}$, H. Marike Boezen ${ }^{8,9}$, \\ Barbara Hoffmann ${ }^{10}$, Susanne Krauss-Etschmann ${ }^{11,12}$, \\ Gerard H. Koppelman (i) $^{9,13}$ and Bertil Forsberg ${ }^{14}$
}

\begin{abstract}
Affiliations: 'Institute of Environmental Medicine, Karolinska Institutet, Stockholm, Sweden. ${ }^{2}$ Sachs' Children's Hospital, Södersjukhuset, Stockholm, Sweden. ${ }^{3}$ Centre for Occupational and Environmental Medicine, Stockholm County Council, Stockholm, Sweden. ${ }^{4}$ INSERM UMR-S1124, Paris, France. ${ }^{5}$ Université

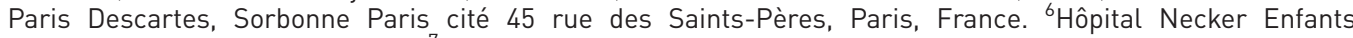
malades, AP-HP, Paris, France. ${ }^{7}$ Advocacy and EU Affairs Dept, European Respiratory Society, Brussels, Belgium. ${ }^{8}$ University of Groningen, University Medical Center Groningen, Dept of Epidemiology, Groningen, The Netherlands. "University of Groningen, University Medical Center Groningen, Groningen Research Institute for Asthma and COPD (GRIAC), Groningen, The Netherlands. ${ }^{10}$ Institute of Occupational, Social and Environmental Medicine, Medical Faculty, Heinrich-Heine University Düsseldorf, Düsseldorf, Germany. ${ }^{11}$ Research Center Borstel, Leibniz-Center for Medicine and Biosciences, Borstel, Germany, Member of the German Center for Lung Research (DZL). ${ }^{12}$ Institute of Experimental Medicine, Christian-Albrechts-University of Kiel, Kiel, Germany. ${ }^{13}$ University of Groningen, University Medical Center Groningen, Beatrix Children's Hospital, Dept of Pediatric Pulmonology and Pediatric Allergology, Groningen, The Netherlands. ${ }^{14}$ Dept of Public Health and Clinical Medicine, Umeå University, Umeå, Sweden.
\end{abstract}

Correspondence: Erik Melén, Institute of Environmental Medicine, Karolinska Institutet, Box 210, SE-171 77 Stockholm, Sweden. E-mail: erik.melenaki.se

@ERSpublications

Time to bridge public health actions for respiratory disease with findings from recent epigenetics research http://ow.ly/iQoD30iyt7I

Cite this article as: Melén E, Barouki R, Barry M, et al. Promoting respiratory public health through epigenetics research: an ERS Environment Health Committee workshop report. Eur Respir J 2018; 51: 1702410 [https://doi.org/10.1183/13993003.02410-2017].

Epigenetics is a rapidly developing research field that is expected to unravel the complex interplay between genes and environmental exposures relevant for health and disease. Epigenetics is classically referred to as heritable phenotypic changes that cannot be explained by changes in DNA sequence. DNA methylation, which is the covalent binding of a methyl group to a cytosine followed by a guanine $(\mathrm{CpG})$, is the most commonly used epigenetic marker in human studies to date. Epigenetics also refers to hydroxymethylation, chromatin remodelling, histone modifications and expression of noncoding RNAs. Recent epidemiological and experimental studies have shown that epigenetic changes are influenced by genetic factors, environmental exposures, ageing and disease processes. In particular, early-life events and exposures seem to strongly influence epigenetic processes such as DNA methylation [1, 2]. From the definition of epigenetics, heritable changes are implied and observations from pre-conceptual exposure studies raise the question whether epigenetic changes really can be inherited at the individual level from one generation to the next [3]. If so, this would have immense public health implications, since generations to come may have to face health consequences of the life their parents and grandparents lived, 
the diseases they had or the environmental factors they were exposed to. There are, however, many question marks around potential epigenetic effects across generations; from epidemiological and experimental evidence to mechanisms and potential health effects. Here, we report on an European Respiratory Society (ERS) Environment and Health Committee workshop held in March 2017 where epigenetics and transgenerational effects in the field of respiratory research were discussed. The aim of the meeting was to examine the role of ERS advocacy in promoting public health policy in relation to epigenetic mechanisms and to identify future research activities.

\section{Environmental exposures and epigenetics}

Both genetic and environmental factors underlie the development of respiratory diseases like asthma and chronic obstructive pulmonary disease (COPD) [4]. Their interaction also plays a role, since individual differences in genetic susceptibility to environmental exposures exist [5, 6]. Besides gene-by-environment interactions, epigenetic mechanisms have been hypothesised as the missing link between the genetic and environmental factors leading to disease [7]. Indeed, over 6000 differential DNA methylation sites have been found associated with in utero and adult cigarette smoking in a variety of studies $[1,8]$. Likewise, differential DNA methylation has also been suggested to have a mediating role in the relation between occupational exposures and the onset of COPD [9]. Given the relatively large proportion of COPD that is related to occupational exposures (up to 50\% [10]), occupational exposures are a major public health concern. Recent population-based genome-wide methylation studies also show that DNA methylation is related to air pollution [11-13]. Air pollution exposures that affect all individuals living in a specific area have direct public health consequences.

\section{The role of epigenetics in respiratory disease}

Epigenetic mechanisms may explain different observations in asthma and COPD, such as environmental effects across generations, parent of origin effects and lifelong effects of early-life exposures. Epigenetics is strongly related to ageing, with thousands of methylation changes observed in whole-blood DNA only in the first 8 years of life [14]. Genes annotated to these CpG sites are enriched for respiratory disease genes, suggesting that dynamic changes in methylation patterns in early life could be related to later respiratory outcomes.

Recently, the epigenetics of asthma was reviewed by YANG et al. [15], stressing the importance of integrating environmental effects on the epigenome and the investigation of relevant cell types (e.g. blood and epithelial cells). As examples, allergic asthma was associated with 81 differential methylation regions in peripheral blood mononuclear cells in inner city asthmatics in the USA, with several immune genes found to be hypomethylated in asthmatic patients, including IL13 and RUNX3 [16]. In a very recent study from the European MeDALL consortium, methylation levels at $14 \mathrm{CpGs}$ were strongly associated with asthma from preschool age to adolescence, but not at birth. Reduced methylation at these CpGs was particularly found in isolated eosinophil cells [17]. Several microRNAs have also been related to asthma and COPD, and importance as a novel therapeutic target has been proposed for both diseases [18, 19]. Changes in gene expression are paralleled by modifications in the histone code, and cell-specific analysis can yield further insights into the interrelation of epigenetic mechanisms and gene transcription [20].

Epigenetic research is now at the forefront of science. However, important questions remain to be answered: are disease-associated epigenetic changes a cause or a consequence of disease? Are tissue or cell-specific epigenetic changes related to disease, and how should we interpret small changes in methylation in relation to gene function? If these epigenetic changes are causally implicated, can these be targeted by novel therapeutics?

\section{Evidence of epigenetic effects across generations and insights from experimental studies}

The epidemiological findings of long-term effects of environmental exposure across several generations are few and inconsistent. Using retrospectively collected smoking information, two studies found an increased risk of asthma in the grandchildren of grandmothers who smoked whilst their mothers were in utero [21, 22]. A third study suggested an increased risk for the daughters of fathers exposed in utero to smoking grandmothers [23]. A recent Swedish study using prospectively collected exposure data found that grandmaternal smoking whilst mothers were in utero was associated with an increased risk of asthma in grandchildren [24].

In experimental studies, exposure to nicotine and tobacco smoke has particularly been investigated for its potential role as an intergenerational (i.e. direct parental effects on the offspring and its germ cells) and transgenerational (i.e. effects on successive generations in the absence of direct exposure of the offspring) risk factor for respiratory diseases [3]. Animal studies have demonstrated the presence of an inherited 
asthma phenotype in the second-generation offspring of nicotine-exposed grandmaternal mice [25]. In this study, the granddams had been given subcutaneous nicotine in a dose equivalent to habitual smokers whilst the first generation (F1) offspring were in utero. In the F2 generation, affected lung function measures were observed, and further studies in the F3 generation also revealed similar lung function changes [26]. Of note, F1 offspring of nicotine-treated dams showed altered DNA methylation and specific modifications of histones ( $\mathrm{H} 3 / \mathrm{H} 4$ acetylation) in both lungs and gonads, implying that epigenetic information could be transmitted via the germline to the F2 generation [25]. Transmission of asthma risk to F2 and F3 generations after exposure to environmental particles during pregnancy has also been observed [27].

So far, inter- and transgenerational studies have primarily focused on disease transmission via the maternal line while paternal spermatozoa were considered as simple transporters of genetic information via their DNA [28]. A recent study, however, observed that paternal smoking also increases the risk of early-onset asthma in offspring [29]. The risk was particularly high when fathers smoked during adolescence, as compared to fathers starting in later life. One mechanistic basis for epigenetic programming via the father is suggested by a study in mice, in which chronic stress altered microRNA signatures in spermatocytes and induced the paternal stress phenotype in offspring [28].

Taken together, there is epidemiological and experimental evidence that parental smoking can affect offspring respiratory health many years later and further, that the risk of lung disease may be passed on to subsequent generations via epigenetic modifications. However, there is a lack of animal studies beyond the F1-F2 generations, which will be needed in order to unravel suggested epigenetic mechanisms underlying transmission of disease risks across generations, and to directly evaluate intervention strategies targeting the epigenome [30].

\section{Public health aspects}

Several reasons, as discussed above, render epigenetics an interesting and potentially very important public health topic. 1) Epigenetic changes may be inheritable, and will thus not only affect the fetus and the germ line in utero, but may also be transmitted via heritable changes to following generations. 2) Epigenetic changes are modifiable and, in principal, reversible, and can therefore be viewed in a similar light as classical modifiable risk factors such as smoking or air pollution. 3) Epigenetics may be particularly important early in life, as evidenced by mechanisms related to embryogenesis and developmental programming. Thus, epigenetic changes have the potential to influence life-long disease occurrence, and their impact might be much larger and longer-lasting than the duration and direct effects of the underlying environmental exposure itself. 4) If epigenetic markers, induced by environmental exposures or other stimuli, distinguish individuals at high risk of later disease, it would enable early, targeted intervention strategies to reduce such risks. 5) From a regulatory point of view, it could also be possible to develop regulatory tests for epigenetic modifications, i.e. epigenotoxicity tests, similar to current available genotoxicity tests.

To better understand the challenges and possibilities that epigenetic research can provide to public health, we recommend the following issues be addressed in future studies: 1) temporal patterns of changes of epigenetic signatures across the life course; 2) changes in epigenetic markers in relation to common exposures, which can vary greatly over time (e.g. air pollution or tobacco smoke exposure); 3 ) the role of epigenetic markers of disease onset and progression, using longitudinal epidemiological studies as well as large-scale epigenetic studies in relevant tissues and cells; and 4) multiple-generation population studies for evaluation of effects across generations. In addition, animal studies supporting evidence for causal effects on the F4 generation are needed.

\section{Concluding remarks}

Medical societies have a key role to play in this debate by remaining strong advocates for public health. The 2017 ERS Presidential Summit noted this need to keep pushing for policies that promote respiratory health through public health measures. Despite advances, there is a need to continue to advocate and educate around risk factors, both known and emerging and provide evidence to policy makers. Policy needs an up-to-date understanding of all risk factors, including those that may influence health in generations to come through epigenetic or other mechanisms. In this article, we have provided arguments for public health concerns related to environmental exposures, epigenetic mechanisms and long-term respiratory health effects. In particular, we recommend that attention should be paid to tobacco smoking and use of nicotine products (including electronic cigarettes), as well as relevant occupational and residential exposures, given the link between these exposures and epigenetic effects. ERS, through its Advocacy Council, Environment and Health Committee, and Tobacco Control Committee, remains committed to delivering this message. 
Support statement: E. Melén is supported by grants from the Swedish Research Council, the Swedish Heart-Lung Foundation and the European Research Council under the European Union (EU) Horizon 2020 (H2020) research and innovation programme (grant agreement number 757919, TRIBAL). R. Barouki is supported by grants from Inserm, Université Paris Descartes, ANR, ANSES, EU FP6 (Heals project) and EU H2020 (HBM4EU). B. Hoffmann is supported by grants from the German Research Council, the Health Effects Institute and the German Environmental Protection Agency (Umweltbundesamt). S. Krauss-Etschmann is supported by grants from the Leibniz Association (Leibniz Competition 2016; Science Campus EvoLung) and the German Lung Research Center (DZL). The funding agencies had no role in writing of the report. Funding information for this article has been deposited with the Crossref Funder Registry.

Conflict of interest: M. Barry is an employee of the European Respiratory Society. G.H. Koppelman has received grants from the Lung Foundation of the Netherlands, TEVA Netherlands, the UBBO EMMIUS Foundation and the TETRI Foundation, outside the submitted work.

\section{References}

1 Joubert BR, Felix JF, Yousefi P, et al. DNA methylation in newborns and maternal smoking in pregnancy: genome-wide consortium meta-analysis. Am J Hum Genet 2016; 98: 680-696.

2 Tobi EW, Goeman JJ, Monajemi R, et al. DNA methylation signatures link prenatal famine exposure to growth and metabolism. Nat Commun 2014; 5: 5592.

3 Krauss-Etschmann S, Meyer KF, Dehmel S, et al. Inter- and transgenerational epigenetic inheritance: evidence in asthma and COPD? Clin Epigenetics 2015; 7: 53.

4 Hobbs $\mathrm{BD}$, de Jong K, Lamontagne $\mathrm{M}$, et al. Genetic loci associated with chronic obstructive pulmonary disease overlap with loci for lung function and pulmonary fibrosis. Nat Genet 2017; 49: 426-432.

5 Gref A, Merid SK, Gruzieva O, et al. Genome-wide interaction analysis of air pollution exposure and childhood asthma with functional follow-up. Am J Respir Crit Care Med 2017; 195: 1373-1383.

6 de Jong K, Vonk JM, Timens W, et al. Genome-wide interaction study of gene-by-occupational exposure and effects on FEV1 levels. J Allergy Clin Immunol 2015; 136: 1664-1672.

7 DeVries A, Vercelli D. Epigenetic mechanisms in asthma. Ann Am Thorac Soc 2016: 13: Suppl. 1, S48-S50.

8 Joehanes R, Just AC, Marioni RE, et al. Epigenetic signatures of cigarette smoking. Circ Cardiovasc Genet 2016; 9: 436-447.

9 van der Plaat DA, de Jong K, de Vries $\mathrm{M}$, et al. Occupational exposure to pesticides is associated with differential DNA methylation. Occup Environ Med 2018 in press [https://doi.org/10.1136/oemed-2017-104787].

10 Bang KM. Chronic obstructive pulmonary disease in nonsmokers by occupation and exposure: a brief review. Curr Opin Pulm Med 2015; 21: 149-154.

11 Gruzieva $\mathrm{O}, \mathrm{Xu} \mathrm{CJ}$, Breton CV, et al. Epigenome-wide meta-analysis of methylation in children related to prenatal $\mathrm{NO}_{2}$ air pollution exposure. Environ Health Perspect 2017; 125: 104-110.

12 Panni T, Mehta AJ, Schwartz JD, et al. Genome-wide analysis of DNA methylation and fine particulate matter air pollution in three study populations: KORA F3, KORA F4, and the Normative Aging Study. Environ Health Perspect 2016; 124: 983-990.

13 de Faria C, van der Plaat D, de Jong K, et al. Long-term air pollution exposure, genome-wide DNA methylation and lung function in the LifeLines cohort study. Environ Health Perspect 2018; 126: 027004.

$14 \mathrm{Xu}$ CJ, Bonder MJ, Soderhall C, et al. The emerging landscape of dynamic DNA methylation in early childhood. BMC Genomics 2017; 18: 25.

15 Yang IV, Lozupone CA, Schwartz DA. The environment, epigenome, and asthma. J Allergy Clin Immunol 2017; 140: $14-23$.

16 Yang IV, Pedersen BS, Liu A, et al. DNA methylation and childhood asthma in the inner city. J Allergy Clin Immunol 2015; 136: 69-80.

$17 \mathrm{Xu}$ C, Söderhäll C, Bustamante $\mathrm{M}$, et al. DNA methylation in childhood asthma: an epigenome-wide meta-analysis. Lancet Respir Med 2018 in press [https://doi.org/10.1016/S2213-2600(18)30052-3].

18 Osei ET, Florez-Sampedro L, Timens W, et al. Unravelling the complexity of COPD by microRNAs: it's a small world after all. Eur Respir J 2015; 46: 807-818.

19 Heffler E, Allegra A, Pioggia G, et al. MicroRNAs profiling in asthma: potential biomarkers and therapeutic targets. Am J Respir Cell Mol Biol 2017; 57: 642-650.

20 Kidd CD, Thompson PJ, Barrett L, et al. Histone modifications and asthma. The interface of the epigenetic and genetic landscapes. Am J Respir Cell Mol Biol 2016; 54: 3-12.

21 Magnus MC, Haberg SE, Karlstad O, et al. Grandmother's smoking when pregnant with the mother and asthma in the grandchild: the Norwegian Mother and Child Cohort Study. Thorax 2015; 70: 237-243.

22 Li YF, Langholz B, Salam MT, et al. Maternal and grandmaternal smoking patterns are associated with early childhood asthma. Chest 2005; 127: 1232-1241.

23 Miller LL, Henderson J, Northstone K, et al. Do grandmaternal smoking patterns influence the etiology of childhood asthma? Chest 2014; 145: 1213-1218.

24 Lodge CJ, Braback L, Lowe AJ, et al. Grandmaternal smoking increases asthma risk in grandchildren: a nationwide Swedish cohort. Clin Exp Allergy 2018; 48: 167-174.

25 Rehan VK, Liu J, Naeem E, et al. Perinatal nicotine exposure induces asthma in second generation offspring. BMC Med 2012; 10: 129.

26 Rehan VK, Liu J, Sakurai R, et al. Perinatal nicotine-induced transgenerational asthma. Am J Physiol Lung Cell Mol Physiol 2013; 305: L501-L507.

27 Gregory DJ, Kobzik L, Yang Z, et al. Transgenerational transmission of asthma risk after exposure to environmental particles during pregnancy. Am J Physiol Lung Cell Mol Physiol 2017; 313: L395-L405.

28 Rodgers $\mathrm{AB}$, Morgan $\mathrm{CP}$, Leu NA, et al. Transgenerational epigenetic programming via sperm microRNA recapitulates effects of paternal stress. Proc Natl Acad Sci USA 2015; 112: 13699-13704.

29 Svanes C, Koplin J, Skulstad SM, et al. Father's environment before conception and asthma risk in his children: a multi-generation analysis of the Respiratory Health In Northern Europe study. Int J Epidemiol 2017; 46: 235-245.

$30 \mathrm{Wu} \mathrm{DD}$, Song J, Bartel S, et al. The potential for targeted rewriting of epigenetic marks in COPD as a new therapeutic approach. Pharmacol Ther 2018; 182: 1-14. 